\title{
Correspondence
}

\section{College Reading Lists}

DeAR SIR

As the compilers of the College Reading Lists in Psychiatry, Part IV, General Reading List, we would like to comment upon Snaith and Baugh's critical letter (Bulletin, July 1981, p. 134).

They 'regret ... that there was considerable reduplication from other lists'. It would be a strange reading list indeed if it amounted only to filling the gaps not covered by the wide array of current specialist reading lists. A general list should cover the best references in all subjects, and the fact that such references were also included in the specialist lists indicated that we and the specialist compilers were in good general agreement-which we found reassuring. It must also be remembered that many trainees may wish to concentrate their efforts on a general reading list which includes the subspecialties rather than consult all of the reading lists.

Your correspondents maintain that the 'enormous labour' in producing the list was 'misdirected', though they do not specify the implied wrong direction of our labours. They describe the list as 'bulky', though-apart from the issue of reduplication-they do not mention what they believe should have been left out. What struck us as odd was their total avoidance of the most conspicuous new feature of the reading list, namely the classification of the literature according to appropriate stages of the life history, and we did wonder whether their remarks implied a disapproval of this approach, which does inevitably involve the inclusion of a number of sections not covered by previous compilers. We would like to emphasize that we regarded this reorientation as our primary objective which we envisaged would put the study of psychiatry into a broader and more interesting perspective.

Perhaps their most central criticism is that our 'updating process has been a failure'. They produce some limited statistics to substantiate this claim. We feel they make the curious and unwarranted assumption that the newest references are the most readworthy and most useful (reminiscent of those patients, and perhaps doctors, who believe the newest drugs to be the most effective). We would contend that updating a reading list involves inserting only those more recent publications which contain significant advances in methodology and results. Had it been our intention, it would not have been difficult for us to restrict our references to those occurring in the late "70s. The reason why 'the proportion appearing later than 1978 was minute' was that our manuscript was submitted to the College towards the end of 1979.

Finally, they assert that 'It should be obvious that no two psychiatrists could be expected to produce a useful list covering the whole field of psychiatry'. They make no refer- ence to the fact that all four previous College general reading lists have been prepared by pairs of psychiatrists. We would maintain that two psychiatrists work well together and that larger numbers of workers run into complications of communication and agreement. As with our predecessors, we drew, where necessary, upon the expertise and experience of those with specialist knowledge.

We were disappointed that Snaith and Baugh's letter did not generate further correspondence. We have received mainly favourable comments from colleagues and trainees, but would be most interested to learn more of the opinions of others.

Graylingwell Hospital

JOHN BIRTCHNELL

Chichester

Saxondale Hospital

Nottingham

EDWARD GORDON

\section{Manpower planuing: Early warning \\ DeAR SiR}

As the bids and approvals for the consultant (manpower) programme for 1982-83 are now known, it has been suggested that we inform the membership of the College of the latest situation and encourage the initiation of moves at local level at this early stage for implementation in the year 198384. A total of 54 consultant bids for the year 1982-83 were received by the Central Manpower Committee (CMC) and approval was given for all 32 in mental illness; all 5 in forensic psychiatry, and 3 in psychotherapy. Only 2 of the 9 bids in mental handicap were allowed, and while the applications in child and adolescent psychiatry were all agreed, they totalled only 5 of the 10-15 possible bids which could have been approved.

Members of the College may not have fully appreciated that a 'shortage specialty' in manpower terms is one in which the anticipated total number of consultant vacancies arising in established posts through retirement or death or in newly created ones cannot be filled by the number of trainees who have completed training. Psychotherapy is not a 'shortage specialty' as although there are few senior registrars, there are even fewer applications for new consultant posts so there are enough trained candidates to fill these and any others vacated. On the other hand, mental handicap, in terms of the definition, is clearly a 'shortage specialty' as, even though there are a greater number of senior registrars, bids for new posts significantly exceed the number of trained psychiatrists available.

As shortage specialties are specifically mentioned in 'Manpower Guidelines', they are certain to get attention at Regional level and thus derive a paradoxical advantage in being categorized in this way. This advantage is borne out by the number of bids received for 'shortage specialties' as against non-shortage specialties in which expansion is not at 